VOL. 75 (2007) [75-89]

\title{
A DEFORMATION OF THE ALEXANDER POLYNOMIALS OF KNOTS YIELDING LENS SPACES
}

\author{
TERUhISA KadoKami AND YUICHI YamadA
}

For a knot $K$ in a homology 3 -sphere $\Sigma$, by $\Sigma(K ; p / q)$, we denote the resulting 3 manifold of $p / q$-surgery along $K$. We say that the manifold or the surgery is of lens type if $\Sigma(K ; p / q)$ has the same Reidemeister torsion as a lens space.

We prove that, for $\Sigma(K ; p / q)$ to be of lens type, it is a necessary and sufficient condition that the Alexander polynomial $\Delta_{K}(t)$ of $K$ is equal to that of an $(i, j)$-torus knot $T(i, j)$ modulo $\left(t^{p}-1\right)$.

We also deduce two results: If $\Sigma(K ; p / q)$ has the same Reidemeister torsion as $L\left(p, q^{\prime}\right)$, then

(1) $q^{\prime}$ or $\overline{q^{\prime}} \equiv \pm q i^{2}(\bmod p)$.

(2) The multiple of $\Delta_{K}\left(t^{k}\right)$ over $k \in\langle i\rangle$ is $\pm t^{m}$ modulo $\left(t^{p}-1\right)$, where $\langle i\rangle$ is the subgroup in $(\mathbf{Z} / p \mathbf{Z})^{\times} /\{ \pm 1\}$ generated by $i$. Conversely, if a subgroup $H$ of $(\mathbf{Z} / p \mathbf{Z})^{\times} /\{ \pm 1\}$ satisfying that the product of $\Delta_{K}\left(t^{k}\right)(k \in H)$ is $\pm t^{m}$ modulo $\left(t^{p}-1\right)$, then $H$ includes $i$ or $j$.

Here, $i, j$ are the parameters of the torus knot above.

\section{INTRODUCTION}

For a knot $K$ in a homology 3 -sphere $\Sigma$, by $\Sigma(K ; p / q)$, we denote the resulting 3 manifold of $p / q$-surgery along $K$. Throughout this paper, we assume that $p \geqslant 2$ and $q \neq 0$ without loss of generality. Then $\Sigma(K ; p / q)$ is a homology lens space whose first homology $H_{1}(\Sigma(K ; p / q) ; \mathbf{Z})$ is isomorphic to the cyclic group $\mathbf{Z} / p \mathbf{Z}$ of order $p$. We study the question "Which $\Sigma(K ; p / q)$ is a lens space?" by algebraic methods.

We let $(\mathrm{Z} / p \mathrm{Z})^{\times}$denote the group, with respect to the multiplication, of invertible elements in the ring $\mathbf{Z} / p \mathbf{Z}$. For an element $i$, by $\bar{i}$ we denote its inverse. In our study, its quotient group $(\mathbf{Z} / p \mathbf{Z})^{\times} /\{ \pm 1\}$ plays an important role. Note that two lens spaces $L\left(p, q_{1}\right)$ and $L\left(p, q_{2}\right)$ are homeomorphic to each other up to orientation if and only if $q_{1}=q_{2}$ or $q_{1}=\overline{q_{2}}$ in $(\mathbf{Z} / p \mathbf{Z})^{\times} /\{ \pm 1\}$.

Received 25th July, 2006

This paper is supported by the 21 COE program "Constitution of wide-angle mathematical basis focused on knots". The second author is partially supported by Grant-in-Aid for Scientific Research No.15740034 and No.18740029, Japan Society for the promotion of Science.

Copyright Clearance Centre, Inc. Serial-fee code: 0004-9727/07 SA2.00+0.00. 
We say that a polynomial $f(t) \in \mathbf{Z}[t]$ is symmetric if $t^{d} f\left(t^{-1}\right)=f(t)$ (thus $f(0) \neq 0$ if $d>0)$, where $d$ is the degree of $f(t)$, or $f(t)=c(\in \mathbf{Z})$. The Alexander polynomial $\Delta_{K}(t)$ is usually defined in the Laurent polynomial ring $\mathbf{Z}\left[t, t^{-1}\right]$ up to multiplication as a unit (a trivial unit) $\pm t^{m}$, where $m$ is an integer. But, in this paper, we always take a regularisation such that $\Delta_{K}(t) \in \mathbf{Z}[t]$, the symmetry $t^{\mathrm{deg}} \Delta_{K}\left(t^{-1}\right)=\Delta_{K}(t)$ holds, and $\Delta_{K}(1)=1$. Here, deg is the degree of $\Delta_{K}(t)$, which is always even. We often use $t^{-(\mathrm{deg} / 2)} \Delta_{K}(t)$ in $\mathrm{Z}\left[t, t^{-1}\right]$.

We often treat with Alexander polynomials in the quotient ring of type $\mathbf{Z}\left[t, t^{-1}\right] / I$, where $I$ is an ideal like $\left(t^{p}-1\right)$. For two elements $f(t), g(t)$ in $\mathbf{Z}\left[t, t^{-1}\right]$ or in $\mathbf{Z}\left[t, t^{-1}\right] / I$, by $f(t) \doteq g(t)$, we denote that $f(t)= \pm t^{m} g(t)$ for an integer $m$.

In $[12,13,14]$, the first author defined the concept "lens type": Roughly speaking, we say that a homology lens space $M$ with $H_{1}(M ; \mathbf{Z}) \cong \mathbf{Z} / p \mathbf{Z}$ is of $(p, q)$-lens type (or simply of lens type) if the Reidemeister torsion of $M$ is same with that of a lens space $L(p, q)$ (for some $q$ ). See Section 2 for the precise definition. Every lens space is of lens type. We adopt the definition of the Reidemeister torsion with a combinatorial "Euler structure" following Turaev $[30,31]$.

Our first main theorem is on a deformation of the Alexander polynomial $\Delta_{K}(t)$ of a knot $K$ in a homology 3-sphere $\Sigma$ which has a lens type surgery, that is, a Dehn surgery yielding a lens type manifold.

THEOREM 1.1. Let $\Delta_{K}(t)$ be the regularised Alexander polynomial of a knot $K$ in a homology 3-sphere $\Sigma$. If a Dehn surgery $\Sigma(K ; p / q)$ is of lens type, then there exist positive integers $i, j$ and a symmetric polynomial $R(t)$ such that the triple $p, i, j$ are mutually coprime, $i j q \equiv \pm 1(\bmod p)$ and $\Delta_{K}(t)$ satisfies

$$
t^{-(\operatorname{deg} / 2)} \Delta_{K}(t)=t^{-((i-1)(j-1)) / 2} \frac{\left(t^{i j}-1\right)(t-1)}{\left(t^{i}-1\right)\left(t^{j}-1\right)}-t^{-(p+1+r) / 2}\left(t^{p}-1\right)(t-1) R(t)
$$

in $\mathbf{Z}\left[t, t^{-1}\right]$, where deg and $r$ are the degrees of $\Delta_{K}(t)$ and $R(t)$, respectively.

Conversely, if $\Delta_{K}(t)$ has such a deformation as above, then $\Sigma(K ; p / q)$ is of lens type, that is, $\Sigma(K ; p / q)$ has the same Reidemeister torsion as that of a lens space.

The first term in the right hand-side of the equality is the Alexander polynomial of the $(i, j)$-torus knot $T(i, j)$ :

$$
\Delta_{T(i, j)}(t)=\frac{\left(t^{i j}-1\right)(t-1)}{\left(t^{i}-1\right)\left(t^{j}-1\right)} .
$$

Thus, for lens surgery along a torus knot in $S^{3}([19]$, see also [12]), Theorem 1.1 holds with $R(t)=0$. The integers $i, j$ in Theorem 1.1 are not unique. Such ambiguity will be considered in Lemma 3.4 in Section 3.

Next, we study the integer $i$ (and $j$ ) that appeared in Theorem 1.1 more, and deduce the following two theorems. 
THEOREM 1.2. If a Dehn surgery $\Sigma(K ; p / q)$ is of lens type, then it is of $\left(p, q i^{2}\right)$ lens type, that is, $\Sigma(K ; p / q)$ has the same Reidemeister torsion as $L\left(p, q i^{2}\right)$, where $i$ is the integer that appeared in Theorem 1.1

Theorem 1.2 extends Fintushel-Stern's in [5, Proposition 1], which says that if a lens space $L(p, r)$ is the integral Dehn surgery on a knot, then $r$ or $-r$ is a quadric residue. As the integer $i$ in Theorem 1.2, we can take the other integer $j$ that appeared in Theorem 1.1. In fact, $i j q \equiv \pm 1$ implies $q j^{2} \equiv \bar{q} \bar{i}^{2}(\bmod p)$ and thus $L\left(p, q j^{2}\right)=L\left(p, q i^{2}\right)$.

THEOREM 1.3. Suppose that $\Sigma(K ; p / q)$ is of lens type as above.

(1) Let $\langle i\rangle$ (and $\langle j\rangle$, respectively) denote the cyclic subgroup in $(\mathbf{Z} / p \mathbf{Z})^{\times} /\{ \pm 1\}$ generated by $i$ (and $j$ ) that appeared in Theorem 1.1. Then, the Alexander polynomial $\Delta_{K}(t)$ of $K$ satisfies

$$
\prod_{k \in\langle i\rangle} \Delta_{K}\left(t^{k}\right) \doteq 1, \quad \text { and } \quad \prod_{k \in\langle j\rangle} \Delta_{K}\left(t^{k}\right) \doteq 1 \quad \text { in } \mathbf{Z}\left[t, t^{-1}\right] /\left(t^{p}-1\right) .
$$

(2) Any subgroup $H$ of $(\mathbf{Z} / p \mathbf{Z})^{\times} /\{ \pm 1\}$ satisfying

$$
\prod_{k \in H} \Delta_{K}\left(t^{k}\right) \doteq 1 \quad \text { in } \mathrm{Z}\left[t, t^{-1}\right] /\left(t^{p}-1\right)
$$

includes $i$ or $j$ (possibly both $i$ and $j$ ).

In [34], the second author announced an example of Theorem 1.3, the case of the famous non-torus lens surgery along the pretzel knot $P(-2,3,7): 18$-surgery $(\langle i\rangle$ $=\{1,5,11\})$ and 19-surgery $(\langle i\rangle=\{1,7,11\})$, see Section 5 .

In some cases, a weaker version of Theorem 1.3 possibly tells us that $\Sigma(K ; p / q)$ is neither of lens type nor a lens space. For this purpose, we state it as a corollary:

COROLlary 1.4. If $\Sigma(K ; p / q)$ is of lens type, then the Alexander polynomial $\Delta_{K}(t)$ of $K$ satisfies

$$
\prod_{k \in(\mathbf{Z} / \mathrm{Z} \mathbf{Z})^{\times} /\{ \pm 1\}} \Delta_{K}\left(t^{k}\right) \doteq 1 \quad \text { in } \mathrm{Z}\left[t, t^{-1}\right] /\left(t^{p}-1\right) .
$$

The paper is organised as follows: In Section 2, we set up the preliminaries and redefine the concept lens type precisely, as a sharpened version of that in the first author's previous papers $[12,13]$. We also review the surgery formula due to Turaev $[29,30$, 31] and Sakai [26], which connects Alexander polynomial and Reidemeister torsion. In Section 3, we prove Theorem 1.1 and 1.2. In Section 4, we prove Theorem 1.3 and study minimal subgroups satisfying the assumption of Theorem 1.3(2). In Section 5, we demonstrate some examples: two examples from rational lens surgeries along torus knots, and the famous non-torus lens surgeries $(P(-2,3,7) ; 18)$ and $(P(-2,3,7) ; 19)$ in $S^{3}$. We also study two families of lens surgery (Fintushel-Stern's knots $K_{n}$ in [5], and Berge's 
knots $k(a, b)$ in $\left.[3,33] ; P(-2,3,7)=K_{2}=k(2,3)\right)$. At the end of the paper, we shall refer to some recent works in this subject.

The authors would like to thank to Professor Eriko Hironaka for informing them on the interest on our work from the view point of "Mahler measure", also Professor Masaaki Ue and Doctor Motoo Tange for their useful advice.

\section{Preliminaries}

In this section, we prepare definitions and notations, and review the results from the first author's results $[\mathbf{1 2}, \mathbf{1 3}]$. Our method is a number theoretic study of Reidemeister torsion in the cyclotomic fields $\mathbf{Q}(\zeta)$. For the definition of Reidemeister torsion, see $[18,29,30,31]$.

2.1. 3-MANIFOLD OF LENS TYPE. For an oriented closed 3-manifold $M$, the concept that $M$ is of lens type was originally defined in $[\mathbf{1 2}, \mathbf{1 3}$, and was applied in [14]. Here we sharpen the definition slightly for the purpose in this paper.

Let $M$ be a homology lens space whose first homology $H_{1}(M ; Z)\left(H_{1}(M)\right.$ for short) is isomorphic to $\mathbf{Z} / p \mathbf{Z}$ generated by an element $t$. We take a ring homomorphism $\psi_{d}$ : $\mathbf{Z}\left[H_{1}(M)\right] \rightarrow \mathbf{Q}(\zeta)$, where the subindex $d(d \geqslant 2)$ is the order of the image $\zeta=\psi_{\mathbf{d}}(t)$ of $t$ (thus $d$ is a divisor of $p$ ), and $\mathbf{Q}(\zeta)$ is the $d$-th cyclotomic field. The Reidemeister torsion $\tau^{\psi_{d}}(M)$ of $M$ associated to $\psi_{d}$ is defined, via the simplicial chain complex of the cyclic Abelian covering space $\widetilde{M}$ as $\mathbf{Z}\left[H_{1}(M)\right]$-module, as an element in $\mathbf{Q}(\zeta)$ up to multiplication by $\pm \zeta^{m}$ for an integer $m$, which we call a trivial unit.

We shall study $\tau^{\psi_{d}}(M)$ of a fixed $M=\Sigma(K ; p / q)$ with various divisors $d$ of $p$. To avoid the ambiguity of the multiplicity of trivial units, we fix an oriented basis of simplicial chain complex of $\widetilde{M}$. Up to now, it has been known that such fixing the basis corresponds to fixing "Euler structure" of the 3-manifold defined by Turaev $([30,31])$, (In such a sense, it might be better that we introduce a new notation such as $\tau^{\psi_{d}}(M, \mathrm{e})$, where, by $\mathbf{e}$, we denote the specified Euler structure, but we keep the shorter notation $\tau^{\psi_{\mathrm{d}}}(M)$.)

Definition 2.1: We say that a homology lens space $M$ with $H_{1}(M) \cong \mathbf{Z} / p \mathbf{Z}$ generated by $t$ is of lens type if there exists $\varepsilon, m, i$ and $j$ such that, for every divisor $d$ of $p(d \geqslant 2)$ and $\psi_{\mathbf{d}}$, the Reidemeister torsion satisfies

$$
\tau^{\psi_{d}}(M)=\varepsilon \zeta^{m}\left(\zeta^{i}-1\right)^{-1}\left(\zeta^{j}-1\right)^{-1}
$$

where $\zeta=\psi_{d}(t)$ is the primitive $d$-th root of unity,

(1) $\varepsilon=1$ or -1 , and $m$ is a positive integer independent from $d$,

(2) $i$ and $j$ are positive integers independent from $d$, and

(3) $i$ and $j$ are coprime to $p$. 
Furthermore, more precisely, a 3-manifold $M$ of lens type is of $(p, q)$-lens type if $i \bar{j} \equiv q$ or $\bar{q}$ in $(\mathbf{Z} / p \mathbf{Z})^{\times} /\{ \pm 1\}$.

The Reidemeister torsion of the lens space $L(p, q)$ is known:

$$
\tau^{\varphi_{d}}(L(p, q))=(\zeta-1)^{-1}\left(\zeta^{\bar{q}}-1\right)^{-1},
$$

for a good choice of $\varphi_{d}$, up to the multiplicity by trivial units. See also the next subsection $(L(p, q)$ is the $p /(-q)$-surgery along the unknot). Each lens space $L(p, q)$ is of $(p, q)$-lens type.

This definition is sharper, at the condition (1), than that in the first author's previous papers $[12,13]$ and the previous joint work $[14]$.

2.2. SURGERY FORMULA FOR REIDEMEISTER TORSIONS. In the case that $M$ is the $p / q$-surgery $\Sigma(K ; p / q)$ on a knot $K$ in a homology 3 -sphere $\Sigma$, we take the oriented meridian of $K$ as the generator $t$ of $H_{1}(M) \cong \mathbf{Z} / p \mathbf{Z}$.

THEOREM 2.2. (Turaev [29]; Sakai [26].) In the case $M=\Sigma(K ; p / q), t$ is a meridian of $K$ and $\psi_{d}(t)=\zeta$, we have

$$
\tau^{\psi_{d}}(M)=\Delta_{K}(\zeta)(\zeta-1)^{-1}\left(\zeta^{\bar{q}}-1\right)^{-1} .
$$

\section{Proofs of TheOREM 1.1 AND 1.2}

Suppose that $\Sigma(K ; p / q)$ is of lens type. Then, by Definition 2.1 and Turaev's theorem 2.2 , it holds that

$$
\Delta_{K}(\zeta)=\varepsilon \zeta^{m} \frac{(\zeta-1)\left(\zeta^{\bar{q}}-1\right)}{\left(\zeta^{i}-1\right)\left(\zeta^{j}-1\right)} \quad \text { in } \mathbf{Z}(\zeta)
$$

for any divisor $d$ of $p$, where $\varepsilon= \pm 1, m, i, j$ ( $i$ and $j$ are coprime to $p$ ) are positive integers, all independent from $d$. In this section, we let $\zeta_{d}$ denote the $d$-th primitive root of unity.

Now, we show the following lemma.

LEMMA 3.1. In the situation above,

(1) $\bar{q} \equiv \varepsilon i j(\bmod p)$, and

(2) $2 m-\operatorname{deg} \equiv-(i-1)(j-1)+(1-\varepsilon) i j(\bmod p)$,

where deg is the degree of the regularised Alexander polynomial $\Delta_{K}(t)$.

PROOF: (1) We took the inverse $\bar{q}$ of $q$ in $(\mathbf{Z} / p \mathbf{Z})^{\times}$before, but here we re-define $\bar{q}$ as a positive integer (a lift) satisfying $q \bar{q} \equiv 1(\bmod p)$. We also fix positive integers $\bar{i}$ and $\bar{j}$ that satisfy $i \bar{i} \equiv j \bar{j} \equiv 1(\bmod p)$. We set

$$
F(t):=\varepsilon t^{m} \frac{t^{i \bar{i}}-1}{t^{i}-1} \cdot \frac{t^{j \bar{j}}-1}{t^{j}-1}
$$


Note that $F(t)$ is a polynomial $(F(t) \in \mathbf{Z}[t])$, because

$$
\frac{t^{k l}-1}{t^{k}-1}=1+t^{k}+t^{2 k}+\cdots+t^{(l-1) k}
$$

for $(k, l)=(i, \bar{i}),(j, \bar{j} \bar{q})$.

By the assumption of lens type-ness (3.1), for each divisor $d$ of $p$ we have $\Delta_{K}\left(\zeta_{d}\right)$ $=F\left(\zeta_{d}\right)$ in $\mathbf{Z}\left[\zeta_{d}\right]$. This implies $\Delta_{K}(t)-F(t)$ is in the kernel of the natural projection $\pi_{d}: \mathbf{Z}[t] \rightarrow \mathbf{Z}\left[\zeta_{d}\right]$ (defined by $\pi_{d}(f(t))=f\left(\zeta_{d}\right)$ ), which is the ideal $\left\langle\Phi_{d}(t)\right\rangle$ generated by the $d$-th cyclotomic polynomial $\Phi_{d}(t)$.

Since cyclotomic polynomials are irreducible and

$$
\prod_{d \mid p, d \geqslant 2} \mathbf{\Phi}_{d}(t)=\frac{t^{p}-1}{t-1}
$$

there exists $g(t) \in \mathbf{Z}[t]$ such that

$$
\Delta_{K}(t)-F(t)=\frac{t^{p}-1}{t-1} \cdot g(t)
$$

By substituting $t=1$, we have

$$
1-\varepsilon \overline{i j} \bar{q}=p \cdot g(1) \equiv 0(\bmod p) .
$$

This means $\bar{q} \equiv \varepsilon i j(\bmod p)$.

(2) By the symmetry of $\Delta_{K}(t)$, we have $\zeta^{\operatorname{deg}} \Delta_{K}\left(\zeta^{-1}\right)=\Delta_{K}(\zeta)$ for the $p$-th primitive root of unity $\zeta$. By Equation (3.1), we have $\zeta^{\operatorname{deg}-m-1-\bar{q}+i+j}=\zeta^{m}$. Here we used $\left(\zeta^{-k}-1\right)=-\zeta^{-k}\left(\zeta^{k}-1\right)$. By the congruence (1), we have the congruence.

Lemma 3.1(1) implies Theorem 1.2;

Proof OF ThEOREM 1.2: If we assume that $\Sigma(K ; p / q)$ is of lens type as Equation (3.1), then the integers $\varepsilon, i, j$ satisfy $\bar{j} \equiv \varepsilon q i(\bmod p)$ by Lemma $3.1(1)$. Thus $i \bar{j}=q i^{2}$ in $(\mathbf{Z} / p \mathbf{Z})^{\times} /\{ \pm 1\}$.

Before starting the proof of Theorem 1.1, we control the indexes $i, j, \varepsilon$ and $m$. First, we may assume the sign $\varepsilon$ is +1 : Because of the equality

$$
\varepsilon \zeta^{m} \frac{(\zeta-1)\left(\zeta^{\varepsilon i j}-1\right)}{\left(\zeta^{i}-1\right)\left(\zeta^{j}-1\right)}=(-\varepsilon) \zeta^{m-i} \frac{(\zeta-1)\left(\zeta^{(-\varepsilon)(-i) j}-1\right)}{\left(\zeta^{(-i)}-1\right)\left(\zeta^{j}-1\right)},
$$

we can change the sign $\varepsilon$ by changing $i$ to $N p-i$ (or $j$ to $N p-j$ ) and $m$, where $N$ is a sufficiently large integer such that $N p-i>0$. Next, we may assume that $i$ and $j$ are coprime (denoted by $(i, j)=1$ ): Because of the prime number theorem of an arithmetic progression, for given integers $i, j$ both coprime to $p$, there exist positive integers $i^{\prime}$ and $j^{\prime}$ such that $i^{\prime} \equiv i, j^{\prime} \equiv j(\bmod p)$ such that $\left(i^{\prime}, j^{\prime}\right)=1$. These changes do not change the class of $i, j$ in $(\mathbf{Z} / p \mathbf{Z})^{\times} /\{ \pm 1\}$. 
Proof of Theorem 1.1: We assume that $(i, j)=1$ and $\varepsilon=1$, by changing the indexes as above, if it is needed. Now, we set

$$
H(t):=t^{m} \frac{(t-1)\left(t^{i j}-1\right)}{\left(t^{i}-1\right)\left(t^{j}-1\right)} \quad \text { in } \mathbf{Z}[t] .
$$

Then, by Lemma $3.1(1)(\bar{q} \equiv i j(\bmod p))$, we have $\Delta_{K}\left(\zeta_{d}\right)=F\left(\zeta_{d}\right)=H\left(\zeta_{d}\right)$ for any divisor $d$ of $p$, and also $\Delta_{K}(1)=H(1)=1$. Thus, by the same argument as in the proof of Lemma 3.1, $\Delta_{K}(t)-H(t)$ is divisible by $\left(t^{p}-1\right)$.

If $p$ is odd, then Lemma $3.1(2)(2 m-\operatorname{deg} \equiv-(i-1)(j-1)(\bmod p))$ implies that $m-(\operatorname{deg} / 2) \equiv-((i-1)(j-1)) / 2(\bmod p)$. This congruence holds even if $p$ is even, but we postpone the proof until the final part, as Claim 3.2. Then,

$$
\left.\mathcal{D}(t):=t^{-(\operatorname{deg} /) 2} \Delta_{K}(t)-t^{-((i-1)(j-1)) / 2} \frac{(t-1)\left(t^{i j}-1\right)}{\left(t^{i}-1\right)\left(t^{j}-1\right)} \quad \text { (in } \mathrm{Z}\left[t, t^{-1}\right]\right)
$$

is also divisible by $\left(t^{p}-1\right)$, that is, there exists $h(t) \in \mathbf{Z}\left[t, t^{-1}\right]$ such that

$$
\mathcal{D}(t)=\left(t^{p}-1\right) \cdot h(t)
$$

Since $\mathcal{D}(t)$ satisfies $\mathcal{D}\left(t^{-1}\right)=\mathcal{D}(t), h(t)$ is divisible by $(t-1)$. Thus we reset $\mathcal{D}(t)$ $=\left(t^{p}-1\right)(t-1) k(t)$, where $k(t) \in \mathbf{Z}\left[t, t^{-1}\right]$. By the symmetry $\mathcal{D}\left(t^{-1}\right)=\mathcal{D}(t)$ again, $k(t)$ has to be in the form required in the statement.

Finally, we prove the following:

Claim 3.2. Even if $p$ is even, $m-(\operatorname{deg} / 2) \equiv-((i-1)(j-1)) / 2(\bmod p)$.

ProOF OF Claim 3.2: If the claimed congruence does not hold, by Lemma 3.1(2), $m-(\operatorname{deg} / 2) \equiv-((i-1)(j-1)+p) / 2(\bmod p)$. Consider

$$
\mathcal{E}(t):=t^{-(\operatorname{deg} / 2)} \Delta_{K}(t)-t^{-((i-1)(j-1)) / 2} \frac{(t-1)\left(t^{i j}-1\right)}{\left(t^{i}-1\right)\left(t^{j}-1\right)}
$$

in $\mathbf{Z}\left[t, t^{-1}\right]$. By the same argument as the proof of Lemma 3.1, we have $\mathcal{E}(t)=0$ in $\mathrm{Z}\left[t, t^{-1}\right] / I_{p / 2}$, where $I_{p / 2}$ is the ideal generated by $\left(t^{p / 2}-1\right)$.

Let $p=2^{r} p^{\prime}$, where $p^{\prime}$ is odd, be the decomposition of $p$. Let $\zeta$ be the $2^{r}$-th primitive root of unity, thus $\zeta^{p / 2}=-1$. Because of the lens type-ness (the indexes has been controlled), we have

$$
\Delta_{K}(\zeta)=\zeta^{m} \frac{(\zeta-1)\left(\zeta^{i j}-1\right)}{\left(\zeta^{i}-1\right)\left(\zeta^{j}-1\right)}
$$

Thus we have

$$
\begin{aligned}
\mathcal{E}(\zeta) & =\zeta^{-(\mathrm{deg} / 2)} \Delta_{K}(\zeta)-\zeta^{-((i-1)(j-1)) / 2} \frac{(\zeta-1)\left(\zeta^{i j}-1\right)}{\left(\zeta^{i}-1\right)\left(\zeta^{j}-1\right)} \\
& =-2 \zeta^{-((i-1)(j-1)) / 2} \frac{(\zeta-1)\left(\zeta^{i j}-1\right)}{\left(\zeta^{i}-1\right)\left(\zeta^{j}-1\right)}
\end{aligned}
$$


and $\mathcal{E}(\zeta) / 2$ has norm $\pm 1:|N(\mathcal{E}(\zeta) / 2)|=1$

On the other hand, because $\mathcal{E}(1 / t)=\mathcal{E}(t)$, there exist $g(t)$ such that $\mathcal{E}(t)=\left(t^{p / 2}\right.$ $-1)(t-1) g(t)$. Substituting $t=\zeta$, we have

$$
\frac{1}{2} \mathcal{E}(\zeta)=\frac{1}{2}\left(\zeta^{p / 2}-1\right)(\zeta-1) g(\zeta)=-(\zeta-1) g(\zeta)
$$

Then, because $|N(\zeta-1)|=2$ and that the norm of an algebraic integer $g(\zeta) \in \mathbf{Z}[\zeta]$ is a non-zero integer, thus $|N(\mathcal{E}(\zeta) / 2)|=2|N(g(\zeta))| \geqslant 2$, . We have a contradiction.

For the norm in a Galois extension over $\mathbf{Q}$, see [32]. The norm of $\Delta_{K}(\zeta)$ in $\mathbf{Q}(\zeta)$ plays an important role in our study, see $[12,14]$.

Corollary 3.3. Let $\Delta_{K}(t)$ be the Alexander polynomial of a knot $K$ in a homology 3-sphere $\Sigma$. If $\Sigma(K ; p / q)$ is of lens type, then there exist positive integers $i, j$ such that the triple $p, i, j$ are mutually coprime, $i j q \equiv \pm 1(\bmod p)$ and $\Delta_{K}(t)$ satisfies

$$
\Delta_{K}(t) \doteq \frac{\left(t^{i j}-1\right)(t-1)}{\left(t^{i}-1\right)\left(t^{j}-1\right)} \quad \text { in } \mathbf{Z}\left[t, t^{-1}\right] /\left(t^{p}-1\right)
$$

We shall use this Corollary in Section 4.

We have already remarked on the non-uniqueness of the indexes $i, j, m$ in Theorem 1.1, but, the theorem is independent of the ambiguity, by the following lemma.

LEMMA 3.4. Let $i$ and $j$ (respectively $i^{\prime}$ and $j^{\prime}$ ) be positive integers such that the triple $p, i, j$ (and $p, i^{\prime}, j^{\prime}$ ) are mutually coprime. We define $H(t)$ and $H^{\prime}(t)$ as follows:

$$
H(t):=t^{-((i-1)(j-1)) / 2} \frac{\left(t^{i j}-1\right)(t-1)}{\left(t^{i}-1\right)\left(t^{j}-1\right)}, \quad H^{\prime}(t):=t^{-\left(\left(i^{\prime}-1\right)\left(j^{\prime}-1\right)\right) / 2} \frac{\left(t^{i^{\prime} j^{\prime}}-1\right)(t-1)}{\left(t^{i^{\prime}}-1\right)\left(t^{j^{\prime}}-1\right)}
$$

(1) If $i \equiv i^{\prime}, j \equiv j^{\prime}(\bmod p)$, then

$$
\frac{(i-1)(j-1)}{2} \equiv \frac{\left(i^{\prime}-1\right)\left(j^{\prime}-1\right)}{2}(\bmod p)
$$

(2) If $i \equiv \pm i^{\prime}, j \equiv \pm j^{\prime}(\bmod p)$, then $H(t)-H^{\prime}(t)$ is divisible by $\left(t^{p}-1\right)$.

Proof: (1) We divide the proof into two cases.

CASE 1. ( $p$ is odd). Then the homomorphism $2: \mathbf{Z} / p \mathbf{Z} \rightarrow \mathbf{Z} / p \mathbf{Z}$ defined by $2(x)=2 x$ is an isomorphism. Thus $i \equiv i^{\prime}, j \equiv j^{\prime}$ implies $(i-1)(j-1) \equiv\left(i^{\prime}-1\right)\left(j^{\prime}-1\right)$ and also the required congruence.

CASE 2. ( $p$ is even). Then both $i$ and $j$ are odd, because of the coprime-ness. We only have to prove the congruence in the case $j=j^{\prime}$. The difference is

$$
\frac{(i-1)(j-1)}{2}-\frac{\left(i^{\prime}-1\right)(j-1)}{2}=\frac{\left(i-i^{\prime}\right)}{2} \cdot(j-1) \equiv 0,
$$

because $(j-1)$ is even. 
(2) First, we treat with the case $i \equiv i^{\prime}, j \equiv j^{\prime}(\bmod p)$. We have $H(1)=H^{\prime}(1)$. By the assumption and the congruence $(1)$, for any divisor $d(\geqslant 2)$ of $p$, we have also $H\left(\zeta_{d}\right)=H^{\prime}\left(\zeta_{d}\right)$. Thus $H(t)-H^{\prime}(t)$ is divisible by $\left(t^{p}-1\right)$.

Next, we treat with the case that $i \equiv-i^{\prime}(\bmod p)$ and $j^{\prime}=j$. By the deformation

$$
t^{-\left(\left(i^{\prime}-1\right)(j-1)\right) / 2} \frac{\left(t^{i^{j} j}-1\right)(t-1)}{\left(t^{i^{\prime}}-1\right)\left(t^{j}-1\right)}=t^{-\left(\left(i^{\prime}-1\right)(j-1)\right) / 2+i^{\prime}(j-1)} \frac{\left(t^{\left(-i^{\prime}\right) j}-1\right)(t-1)}{\left(t^{\left(-i^{\prime}\right)}-1\right)\left(t^{j}-1\right)} .
$$

and that

$$
-\frac{\left(i^{\prime}-1\right)(j-1)}{2}+i^{\prime}(j-1)=-\frac{\left(\left(-i^{\prime}\right)-1\right)(j-1)}{2} \equiv-\frac{(i-1)(j-1)}{2}
$$

by (1), we can go back to the previous case. The other cases can be proved by combination of the cases above.

\section{Proof of Theorem 1.3}

First, we remark that, if $q=1$ (that is, the case of integral surgery), then $\langle i\rangle=\langle j\rangle$, because we have $j= \pm \bar{i}$ by Lemma 3.1(1). On the other hand, in the case of rational surgery, there exists a case that $\langle i\rangle \neq\langle j\rangle$ (possibly $\langle i\rangle \subset\langle j\rangle,\langle j\rangle \subset\langle i\rangle$, or $(\langle i\rangle \not \subset\langle j\rangle$ and $\langle i\rangle \not \supset\langle j\rangle))$, see examples in Section 5.

Proof of (1): Suppose that $\Sigma(K ; p / q)$ is of lens type. By Corollary 3.3 , there exist positive integers $i, j$ and an integer $m$ (according to the indexes of the lens type-ness), such that

$$
\Delta_{K}(t)=t^{m} \frac{\left(t^{i j}-1\right)(t-1)}{\left(t^{i}-1\right)\left(t^{j}-1\right)} \text { in } \mathbf{Z}\left[t, t^{-1}\right] /\left(t^{p}-1\right) .
$$

Because of the symmetry between $i$ and $j$, we only prove the theorem in the case of $i$. We let $I$ denote the order of $i$ in the group $(Z / p Z)^{\times} /\{ \pm 1\}$, thus $I$ is equal to the cardinality of the subgroup $\langle i\rangle$. We shall take the multiple of $\Delta_{K}\left(t^{k}\right)$ 's over $k \in\langle i\rangle$. If $k^{\prime} \equiv k(\bmod p)$, any polynomial $f(t)$ satisfies $f\left(t^{k^{\prime}}\right) \equiv f\left(t^{k}\right)\left(\bmod \left(t^{p}-1\right)\right)$. Our regularised $\Delta_{K}(t)$ satisfies $\Delta_{K}\left(t^{-k}\right)=t^{-k \cdot \operatorname{deg}} \Delta_{K}\left(t^{k}\right)$. Thus, we identify $\langle i\rangle$ (as a subset of $\left.(\mathbf{Z} / p \mathbf{Z})^{\times} /\{ \pm 1\}\right)$ with the set $\left\{1, i, i^{2}, \ldots, i^{I-1}\right\}$ in $\mathbf{Z}$ naturally. We define an integer $M$ by $M:=m\left(1+i+i^{2}+\cdots+i^{I-1}\right)$.

By the fractional reductions, it holds that, modulo $\left(t^{p}-1\right)$,

$$
\begin{aligned}
\prod_{k \in\langle i\rangle} \Delta_{K}\left(t^{k}\right) & =t^{M} \frac{\left(t^{i j}-1\right)(t-1)}{\left(t^{i}-1\right)\left(t^{j}-1\right)} \cdot \frac{\left(t^{i^{2} j}-1\right)\left(t^{i}-1\right)}{\left(t^{i^{2}}-1\right)\left(t^{i j}-1\right)} \ldots \\
& =t^{M} \frac{(t-1)\left(t^{i^{I} j}-1\right)}{\left(t^{j}-1\right)\left(t^{i^{I}}-1\right)} .
\end{aligned}
$$

Now, we set a polynomial $F(t)$ by

$$
F(t):= \begin{cases}t^{M} & \text { if } i^{I}=1 \text { in }(\mathbf{Z} / p \mathbf{Z})^{\times} \\ t^{M-j+1} & \text { if } i^{I}=-1 \text { in }(\mathbf{Z} / p \mathbf{Z})^{\times} .\end{cases}
$$


Then, for any divisor $d$ of $p$, it is easy to show

$$
\prod_{k \in(i)} \Delta_{K}\left(\zeta_{d}^{k}\right)=F\left(\zeta_{d}\right)
$$

where $\zeta_{d}$ is the $d$-th primitive root of unity, and $F(1)=1$. By the same argument with in the proof of Lemma 3.1 and Theorem 1.1, we have the conclusion.

Before starting the proof of Theorem 1.3(2), we recall Franz's number-theoretical lemma ([6]), which was used in the classification of lens spaces, see also $[12,14]$. It says that there is no relation of a special fractional type among the elements of $\left(\zeta^{x}-1\right)^{ \pm 1}$ type in $\mathbf{Q}(\zeta)$.

LEMMA 4.1. (Franz [6]) Let $\zeta$ be a primitive $n$-th root of unity. Let $a_{i}$ ( $i \in$ $\left.(\mathbf{Z} / n \mathbf{Z})^{\times}\right)$be an integer satisfying the following conditions:

(1) $a_{-i}=a_{i}$,

(2) $\sum_{i \in(\mathbf{z} / n \mathbf{Z})^{\times}} a_{i}=0$,

(3) $\prod_{i \in(\mathbf{Z} / n \mathbf{Z}) \times}\left(\zeta^{i}-1\right)^{a_{i}}=1$.

Then $a_{i}=0$ for all $i \in(\mathbf{Z} / n \mathbf{Z})^{\times}$.

Proof of (2): Suppose that a subgroup $H$ of $(\mathbf{Z} / p \mathbf{Z})^{\times} /\{ \pm 1\}$ satisfies that

$$
\prod_{k \in H} \Delta_{K}\left(t^{k}\right) \doteq 1 \quad \text { in } \mathbf{Z}\left[t, t^{-1}\right] /\left(t^{p}-1\right)
$$

Let $\zeta$ be the $p$-th primitive root of unity. By Corollary 3.3,

$$
\prod_{k \in H} \Delta_{K}\left(\zeta^{k}\right)=\frac{\prod_{k \in H}\left(\zeta^{k i j}-1\right) \cdot \prod_{k \in H}\left(\zeta^{k}-1\right)}{\prod_{k \in H}\left(\zeta^{k i}-1\right) \cdot \prod_{k \in H}\left(\zeta^{k j}-1\right)} \doteq 1
$$

This equation and Franz's lemma implies (see $[\mathbf{1 2}, \mathbf{1 4}])$ :

$$
\{k i j, k(k \in H)\}=\{k i, k j(k \in H)\} \quad \text { in }(\mathbf{Z} / p \mathbf{Z})^{\times} /\{ \pm 1\}
$$

that is, $i j H \cup H=i H \cup j H$, where $x H$ is the coset of the group $(\mathbf{Z} / p \mathbf{Z})^{\times} /\{ \pm 1\}$ by $H$. Thus, it holds that either $H \ni i$ or $i j H \ni i$ (thus $H \ni j$ ). Possibly both of them hold. We have the theorem.

Since $H$ is a subgroup, $H \ni i$ implies $H \supset\langle i\rangle$. Thus, on the minimal subgroups $H$ (with respect to the inclusion relation) in $(Z / p Z)^{\times} /\{ \pm 1\}$ that satisfy

$$
\prod_{k \in H} \Delta_{K}\left(t^{k}\right) \doteq 1 \quad \text { in } \mathbf{Z}\left[t, t^{-1}\right] /\left(t^{p}-1\right)
$$

we have the following: 
COROLlary 4.2. Let $K$ be a knot in a homology 3-sphere $\Sigma$ with the Alexander polynomial $\Delta_{K}(t)$, and suppose that $\Sigma(K ; p / q)$ is of lens type. If a subgroup $H$ is minimal among the subgroups in $(\mathbf{Z} / p \mathbf{Z})^{\times} /\{ \pm 1\}$ that satisfy the equation (4.2), then it holds that $H=\langle i\rangle$ or $H=\langle j\rangle$ (possibly both of them hold).

Thus, the number of minimal subgroups $H$ of $(\mathbf{Z} / p \mathbf{Z})^{\times} /\{ \pm 1\}$ that satisfy the equation (4.2) is at most two.

By using Corollary 4.2, in some cases, for given $\Delta_{K}(t)$ and $p$, we can prove that $\Sigma(K ; p / q)$ is not of lens type for any $q$ :

EXAMPLE 4.3. Let $K$ be a knot whose Alexander polynomial is the 429-th cyclotomic polynomial $(429=3 \cdot 11 \cdot 13)$ :

$$
\Delta_{K}(t)=\frac{\left(t^{429}-1\right)\left(t^{13}-1\right)\left(t^{11}-1\right)\left(t^{3}-1\right)}{\left(t^{143}-1\right)\left(t^{39}-1\right)\left(t^{33}-1\right)(t-1)}
$$

and the coefficient $61 / q$. In this case, in the group $(\mathbf{Z} / 61 \mathbf{Z})^{\times} /\{ \pm 1\}(\cong \mathbf{Z} / 30 \mathbf{Z}$, generated by 2$)$, there are three distinct minimal subgroups: $\langle 3\rangle$ of order $5,\langle 11\rangle$ of order 2 , and $\langle 13\rangle$ of order 3 , and all of them satisfy the equation (4.2). Thus, any $(K ; 61 / q)$ is not of lens type.

\section{EXAMPLES}

First, we demonstrate two examples of lens surgery along the torus knot ([19]) with rational coefficient.

EXAMPLE 5.1. Let $K$ be the torus $\operatorname{knot} T(2,3)$ in $S^{3}$ and $p / q:=17 / 3$. Then $(K ; p / q)$ is the lens space $L(17,5)$. We can take $i=2, j=3$ as the indexes. In this case, in the group $(\mathbf{Z} / \mathbf{1 7 Z})^{\times} /\{ \pm 1\}(\cong \mathbf{Z} / 8 \mathbf{Z}$, generated by 3$)$, it holds that $\langle i\rangle \neq\langle j\rangle$ and $\langle i\rangle \subset\langle j\rangle$.

EXAMPLE 5.2. Let $K$ be the torus $k$ not $T(2,5)$ in $S^{3}$ and $p / q:=31 / 3$. Then $(K ; p / q)$ is the lens space $L(31,18)$. We can take $i=2, j=5$ as the indexes. In this case, in the group $(\mathbf{Z} / 31 \mathbf{Z})^{\times} /\{ \pm 1\}(\cong \mathbf{Z} / 15 \mathbf{Z}$, generated by 3 ). The order of $\langle i\rangle$ (or $\langle j\rangle)$ is 5 (or 3 , respectively)), it holds that $\langle i\rangle \neq\langle j\rangle$ and $\langle i\rangle,\langle j\rangle \neq(\mathbf{Z} / 31 \mathbf{Z})^{\times} /\{ \pm 1\}$.

Next, we study the famous lens surgery along non-torus knots:

EXAMPLE 5.3. ([34]) 18-surgery (and 19-surgery also) along the pretzel knot $P(-2,3,7)$ is the lens space $L(18,5)$ (and $L(19,-7)$, respectively)([5]). We let $\Delta_{P}(t)$ denote the Alexander polynomial of $P(-2,3,7)$ :

$$
\Delta_{P}(t)=t^{10}-t^{9}+t^{7}-t^{6}+t^{5}-t^{4}+t^{3}-t+1
$$

It holds that

$$
\Delta_{P}(t) \cdot \Delta_{P}\left(t^{5}\right) \cdot \Delta_{P}\left(t^{11}\right)=t^{13} \quad \text { in } \mathbf{Z}[t] /\left(t^{18}-1\right)
$$


corresponding to $(\mathbf{Z} / 18 \mathrm{Z})^{\times} /\{ \pm 1\} \cong \mathbf{Z} / 3 \mathbf{Z}$ (generated by 5 ). And it holds that

$$
\Delta_{P}(t) \cdot \Delta_{P}\left(t^{7}\right) \cdot \Delta_{P}\left(t^{11}\right)=1 \quad \text { in } \mathrm{Z}[t] /\left(t^{19}-1\right) .
$$

In this case $(p=19)$, in $(\mathbf{Z} / 19 \mathbf{Z})^{\times} /\{ \pm 1\}(\cong \mathbf{Z} / 9 \mathbf{Z}$, generated by 3$), 7$ generates the minimal cyclic subgroup of order 3 .

Note that $\Delta_{P}(-t)$ is known as Lehmer's polynomial in the number theory, see [8, $16,27]$.

The former, 18-surgery along $P(-2,3,7)$ (as $K_{2}$ ) belongs to the family $K_{n}$ of lens surgery found by Fitushel and Stern in [5]. The latter, 19-surgery (as $k(2,3))$ belongs to the family $k(a, b)$ of lens surgery $([\mathbf{1}, \mathbf{3}]$, see also [33]). Recently, Baker named this family TypeVII in [2]. Here, we study the Alexander polynomial of them.
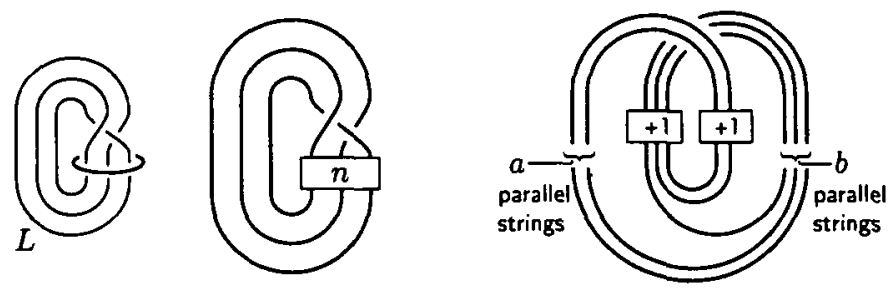

Figure 1: $K_{n}$ and $k(a, b) \quad\left(K_{2}=k(2,3)=P(-2,3,7)\right)$

EXAMPLE 5.4. Let $K_{n}$ be a knot in Figure 1, where $n$ is an integer with $n \geqslant 2$, and the rectangle with $n$ means the $n$ times right full-twist. $9 n$-surgery along $K_{n}$ is a lens space $-L(9 n, 3 n+1)$. Note that

$$
-L(9 n, 3 n+1)=L(9 n, 6 n-1)=-L(9 n, 6 n+1)=L(9 n, 3 n-1) .
$$

The Alexander polynomial $\Delta_{K_{n}}(t)$ of $K_{n}$ is:

$$
\Delta_{K_{n}}(t)=t^{3 n-1}+\frac{\left(t^{3 n}-1\right)^{2}(t-1)}{t^{3}-1} .
$$

The order is $2(3 n-1)$. (The authors first calculated the two-variable polynomial $\Delta_{L}\left(t_{1}, t_{2}\right)$ by using the method in $[10,11]$, and got $\Delta_{K_{n}}(t)$.)

In this case, we can deform $\Delta_{K_{n}}(t)$ as

$$
t^{-(3 n-1)} \Delta_{K_{n}}(t)=t^{-9 n^{2}} \frac{\left(t^{(3 n+1)(6 n+1)}-1\right)(t-1)}{\left(t^{3 n+1}-1\right)\left(t^{6 n+1}-1\right)}+t^{*}\left(t^{9 n}-1\right)(t-1) f(t),
$$

where $f(t)$ is a symmetric polynomial and the index $*$ is decided as in Theorem 1.1. It holds

$$
\Delta_{K_{n}}(t) \cdot \Delta_{K_{n}}\left(t^{3 n+1}\right) \cdot \Delta_{K_{n}}\left(t^{6 n+1}\right)=t^{-3} \quad \text { in } \mathbf{Z}\left[t, t^{-1}\right] /\left(t^{9 n}-1\right) .
$$

In $(Z / 9 n Z)^{\times} /\{ \pm 1\},(3 n+1)$ generates the minimal cyclic subgroup of order 3 . 
EXAMPLE 5.5. Let $k(a, b)$ be the knot in Figure 1, where $(a, b)$ is a pair of coprime positive integers. It is known that $\left(a^{2}+a b+b^{2}\right)$-surgery along $k(a, b)$ is a lens space $-L\left(a^{2}+a b+b^{2}, a \bar{b}\right)$. Note that $(a \bar{b})^{3}=1$ in $\left(\mathbf{Z} /\left(a^{2}+a b+b^{2}\right) \mathbf{Z}\right)^{\times}$, because $a^{3} \equiv b^{3}$ $\bmod \left(a^{2}+a b+b^{2}\right)$.

In the case $(a, b)=(2,2 n+1)$, the coefficient is $a^{2}+a b+b^{2}=4 n^{2}+8 n+7$, and

$$
a \bar{b}=2 n^{2}+3 n+2, \quad(a \bar{b})^{2}=2 n^{2}+5 n+4 .
$$

We set

$$
(i, j)=\left\{\begin{array}{ll}
\left(2 n^{2}+3 n+2,2 n^{2}+5 n+4\right) & \text { if } n \text { is odd } \\
\left(2 n^{2}+5 n+5,2 n^{2}+3 n+3\right) & \text { if } n \text { is even }
\end{array} .\right.
$$

Then the pair $(i, j)$ becomes coprime. By some calculus, we get

$$
\Delta_{k(2,2 n+1)}(t)=\frac{(t-1)\left\{\begin{array}{r}
\left(t^{2 n^{2}+5 n+4}+1\right)\left(t^{(2 n+1)(n+1)}-1\right) \\
+t^{2 n^{2}+3 n+2}\left(t^{2 n+1}-1\right)
\end{array}\right\}}{\left(t^{2 n+1}-1\right)\left(t^{2 n+3}-1\right)} .
$$

It satisfies

$$
\Delta_{k(2,2 n+1)}(t) \cdot \Delta_{k(2,2 n+1)}\left(t^{i}\right) \cdot \Delta_{k(2,2 n+1)}\left(t^{j}\right) \doteq 1
$$

$\bmod \left(t^{4 n^{2}+8 n+7}-1\right)$. See [34] in the case $n=2$.

The authors conjecure that for any coprime positive pair $(a, b)$, for the cubic root $\omega=a \bar{b}$ in $\left(\mathbf{Z} /\left(a^{2}+a b+b^{2}\right) \mathbf{Z}\right)^{\times} /\{ \pm 1\}$,

$$
\Delta_{k(a, b)}(t) \cdot \Delta_{k(a, b)}\left(t^{\omega}\right) \cdot \Delta_{k(a, b)}\left(t^{\omega^{2}}\right) \doteq 1 \bmod \left(t^{a^{2}+a b+b^{2}}-1\right) .
$$

This conjecture is true in the cases that $a^{2}+a b+b^{2}$ is a power of an odd prime. It is proved by Theorem 1.2 and that $\left(Z /\left(a^{2}+a b+b^{2}\right) \mathbf{Z}\right)^{\times} /\{ \pm 1\}$ is a cyclic group.

For other lens surgeries, see Berge's work $[3,4]$, and $[\mathbf{1}, \mathbf{2}, \mathbf{1 7}]$. For researches on Alexander polynomials of knots from the algebraic view points, see $[7,20,23]$ and so on.

On the recent researches. In [9], Ichihara, Saito and Teragaito calculated the Alexander polynomials of knots in homology 3-spheres yielding $L(p, q)$ which include Berge's knots in $S^{3}$. Their form of the Alexander polynomial includes three parameters $p, q$ and $u$ (satisfying $q \equiv \pm u^{2}(\bmod p)$ ), which is derived from the "dual knot" $K^{*}$ in $L(p, q)$ $([3,24])$. Then we raise a problem.

Problem 5.6. Calculate the Alexander polynomial of a Berge's knot in $S^{3}$ with Berge's original parameters in [4] itself.

Recently in [28], Tange reproved our Theorem 1.1 by using the "correction term invariant" due to Ozsváth and Szabó $[15,21,22]$.

Problem 5.7. Are Saito's parameter $u$ in $[\mathbf{2 4}, \mathbf{2 5}]$, Tange's parameter $h$ in $[\mathbf{2 8}]$, and Berge's parameter $B$ in [4], regarded as in $(Z / p Z)^{\times} /\{ \pm 1\}$, always equal to our parameter $i$ or $j$ for general $p$ (the coefficients of the lens surgeries)? 


\section{REFERENCES}

[1] K. Baker, Knots on once-punctured torus fibers, Dissertation (The university of Texas Austin, 2004).

[2] K. Baker, 'Surgery descriptions and volumes of Berge knots I: Large volume Berge knots', arXiv: math.GT/0509054.

[3] J. Berge, 'Some knots with surgeries yielding lens spaces', (Unpublished manuscript, 1990).

[4] J. Berge, "The knots in $D^{2} \times S^{1}$ which have nontrivial Dehn surgeries that yield $D^{2} \times S^{1}$, Topology Appl. 38 (1991), 1-19.

[5] R. Fintushel and R.J. Stern, 'Constructing lens spaces by surgery on knots', Math. $Z$. 175 (1980), 33-51.

[6] W. Franz, 'Über die Torsion einer Überdeckung', J. Reine Angew. Math. 173 (1935), 245-254.

[7] F. González Acuña and H. Short, 'Cyclic branched coverings of knots and homology spheres', Rev. Mat. Univ. Complut. Madrid 4 (1991), 97-120.

[8] E. Hironaka, 'The Lehmer polynomial and pretzel links', Canad. Math. Bull. 44 (2001), $440-451$.

[9] K. Ichihara, T. Saito and M. Teragaito, 'Alexander polynomials of doubly primitive knots', Proc. of Amer. Math. Soc. 135 (2007), 605-615.

[10] T. Kadokami, Seifert complex for links and 2-variable Alexander matrices, (Shin'ichi Suzuki, Editor), Proceedings of Knots'96 (World Sci. Publising, River Edge, 1997), pp. 395-409.

[11] T. Kadokami, On 2-component links with trivial components, Proceedings of Applied Mathematics Workshop 8, The 5th Korea-Japan School of Knots and Links (Center for Applied Mathematics, KAIS, Taejon, Korea, 1997), pp. 71-93.

[12] T. Kadokami, 'Reidemeister torsion and lens surgeries on knots in homology 3-spheres I', Osaka J. Math 43 (2006), 1-15.

[13] T. Kadokami, 'Reidemeister torsion and lens surgeries on knots in homology 3-spheres II', (preprint (2005)).

[14] T. Kadokami and Y. Yamada, 'Reidemeister torsion and lens surgeries on $(-2, m, n)$-pretzel knots', Kobe J. Math. 23 (2006), 65-78.

[15] P.B. Kronheimer, T.S. Mrowka P. Ozsváth and Z. Szabó, 'Monopoles and lens space surgeries', arXiv: math.GT/0310164.

[16] D.H. Lehmer, 'Factorization of certain cyclotomic functions', Ann. of Math. (2) 34 (1933), 461-479.

[17] N. Maruyama, 'On Dehn surgery along a certain family of knots', J. Tsuda College 19 (1987), 261-280.

[18] J. Milnor, 'A duality theorem for Reidemeister torsion', Ann. of Math. (2) 76 (1962), $137-147$.

[19] L. Moser, 'Elementary surgery along a torus knot', Pacific J. Math. 38 (1971), 737-745.

[20] A. Noguchi, 'Zeros of the Alexander polynomial of knot', (preprint (2003)).

[21] P. Ozsváth and Z. Szabó, 'Absolutely graded Floer homologies and intersection forms for four-manifolds with boundary', Adv. Math. 173 (2003), 179-261. 
[22] P. Ozsváth and Z. Szabó, 'On knot Floer homology and lens space surgeries', Topology 44 (2005), 1281-1300.

[23] R. Riley, 'Growth of order of homology of cyclic branched covers of knots', Bull. London Math. Soc. 22 (1990), 287-297.

[24] T. Saito, 'Dehn surgery and (1,1)-knots in lens space', Topology Appl. (to appear).

[25] T. Saito, 'The dual knots of doubly primitive knots', (preprint (2004)).

[26] T. Sakai, 'Reidemeister torsion of a homology lens space', Kobe J. Math. 1 (1984), 47-50.

[27] D. Silver and S. Williams, 'Mahler measure, links and and homology growth', Topology 41 (2002), 979-991.

[28] M. Tange, 'Ozsváth Szabó's correction term and lens surgery', (preprint (2005)).

[29] V.G. Turaev, 'Reidemeister torsion in knot theory', Russian Math. Surveys 41 (1986), 119-182.

[30] V.G. Turaev, Introduction to combinatorial torsions Notes taken by Felix Schlenk, Lectures in Mathematics ETH Zürich (Birkhäuser Verlag, Basel, 2001).

[31] V.G. Turaev, Torsions of 3-dimensional Manifolds, Progress in Mathematics 208 (Birkhäuser Verlag, Basel, 2002).

[32] L.C. Washington, Introduction to cyclotomic fields, Graduate Texts in Mathematics 83 (Springer-Verlag, New York, 1982).

[33] Y. Yamada, 'Berge's knots in the fiber surfaces of genus one, lens spaces and framed links', J. Knot Theory Ramifications 14 (2005), 177-188.

[34] Y. Yamada, 'Two equalities on the Alexander polynomial of the pretzel knot of type $(-2,3,7)^{\prime}$, Bull. Univ. Electro-Comm. 18-1.2 (2006), 47-52.

Department of Mathematics

Osaka City University

Sugimoto3-3-138, Sumiyoshi-ku

Osaka 558-8585

Japan

e-mail: kadokami@sci.osaka-cu.ac.jp
Department of Systems Engineering

The University of Electro-Communications

1-5-1 Chofu-gaoka, Chofu

tokyo $182-8585$

Japan

e-mail: yyyamada@sugaku.e-one.uec.ac.jp 